\title{
Endovascular Treatment of Intracranial Dural Arteriovenous Fistulas: A German Single-Center Experience
}

\author{
Volker Maus ${ }^{a} \quad$ Finn Drescher ${ }^{a} \quad$ Lukas Goertz $^{b} \quad$ Anushe Weber $^{a}$ \\ Werner Weber ${ }^{a} \quad$ Sebastian Fischer ${ }^{a}$ \\ anstitute of Diagnostic and Interventional Radiology, Neuroradiology and Nuclear \\ Medicine, Universitätsklinikum Knappschaftskrankenhaus Bochum, Bochum, Germany; \\ ${ }^{b}$ Department of Neurosurgery, University Hospital of Cologne, Cologne, Germany
}

\section{Keywords}

Dural fistulas · Vascular radiology · Vascular malformations · Endovascular interventional neuroradiology

\begin{abstract}
Background and Purpose: Intracranial dural arteriovenous fistulas (DAVFs) are abnormal shunts between dural arteries and dural venous sinus or cortical veins. We report our experience with endovascular therapy of primary complex DAVFs using modern embolic agents. Methods: This is a retrospective analysis of patients with DAVFs treated between 2015 and 2019. Patient demographics and technical aspects including the use of embolic agent, access to the fistula, number of treatments, occlusion rates, and complications were addressed. Angiographic treatment success was defined as complete occlusion (CO) of the DAVF. Results: Fifty patients were treated endovascularly. Median age was 61 years and $66 \%$ were men. The most common symptom was pulsatile tinnitus in 17 patients (34\%). The most frequent location of the DAVF was the transverse-sigmoid sinus (40\%). Thirty-six fistulas (72\%) had cortical venous reflux. Nonadhesive and adhesive liquid agents were used in $92 \%$ as a single material or in combination. CO was achieved in 48 patients (96\%). In 28 individuals (56\%), only 1 procedure was necessary. Nonadhesive liquid agents were exclusively used in 14 patients (28\%) with $\mathrm{CO}$ attained in every case. For CO of tentorial DAVFs, multiple sessions were more often required than at the other locations ( 55 vs. $14 \%, p=0.0051$ ). Among 93 procedures, the overall complication rate was $3 \%$. The procedure-related mortality rate was $0 \%$. Conclusion: Endovascular treatment of intracranial DAVFs is feasible, safe, and effective with high rates of CO. In more than half of the patients, the DAVF was completely occluded after a single procedure. However, in tentorial DAVFs, multiple sessions were more often required.
\end{abstract}


Maus et al.: Endovascular Treatment of Intracranial DAVFs: A German Single-Center Experience

\section{Introduction}

Intracranial dural arteriovenous fistulas (DAVFs) are abnormal acquired direct shunts between the dural arteries and dural venous sinus or cortical veins, and they account for $10-15 \%$ of all intracranial vascular malformations [1]. DAVF etiology is not yet fully understood, but it is thought they are associated with sinus thrombosis, trauma, tumors, and previous intracranial surgery [2]. Clinical symptoms include hemorrhagic and nonhemorrhagic manifestations and are dependent on the location, flow pattern, and venous pressure conditions [3-5]. For the classification of DAVFs, the system of Cognard et al. [6] and Borden et al. [7] is widely used and is based on the type of venous drainage. The overall risk of intracranial hemorrhage (ICH) from a DAVF is approximately $1.5 \%$ per year, but this increases to up to $19 \%$ in individuals with venous ectasia [8] and a rebleeding rate of $35 \%$ within the first 2 weeks after the initial event $[9,10]$. This highlights the importance of state-of-the-art treatment by an interdisciplinary neurovascular team. In patients with cortical venous reflux (CVR), in particular, acute ICH, or in otherwise severely affected individuals, occlusion of the dural shunts is mandatory.

For the majority of DAVFs, transarterial and/or transvenous embolization is nowadays considered the first-line therapy. The development of catheters and embolization techniques and agents has advanced in the last 2 decades, providing efficient fistula embolization with a minimally invasive approach [11-16]. The first steps were already made in the eighties when intracranial vascular malformations were treated with acryl glue [17], e.g., in combination with transvenous coil occlusion [18]. Until now, the adhesive liquid embolic agent $n$-butyl cyanoacrylate (NBCA) has retained significance for the treatment of DAVFs. New treatment approaches were enabled with an increase in the proportion of cases treated via the arterialonly route [19].

In this study, we present our single-center experience of patients suffering from intracranial DAVFs treated with transarterial and/or transvenous embolization with curative intent, and we also discuss the current endovascular treatment options.

\section{Materials and Methods}

This is a retrospective analysis of patients with cranial DAVFs treated endovascularly in our institution between January 2015 and August 2019. Patients with spinal DAVFs, an age $<18$ years, and individuals with surgery as the primary treatment were excluded. Patient demographics, clinical symptoms, and angiographic characteristics of DAVF (on proceduraland follow-up angiogram) were analyzed by 2 independentreaders. Furthermore, the use of an embolic agent, access to the fistula, and peri- and postinterventional complications were examined. The essential features of each DAVF were assessed, including fistula localization, feeding arteries, and drainage pattern with the occurrence of CVR according to the classification of Cognard et al. [6] and Borden et al. [7]. Fistulas with existing CVR (Cognard $\geq$ IIB) were defined as "malignant DAVFs." All indications were based on interdisciplinary decisions by neurosurgeons and interventional neuroradiologists. In patients with ICH and evidence of hydrocephalus, external ventricular or lumbar drainage was performed prior to the endovascular treatment. Angiographic treatment success was defined as complete closure of the DAVF and an absence of early filling of its venous drainage, even spontaneously during angiographic follow-up after DAVF "down-sizing." In cases when residual arteriovenous shunts were detectable, treatment success was considered incomplete. The statistical significance of observed differences between groups was assessed with the Student $t$ test or $\chi^{2}$ test, when appropriate. All calculations were performed

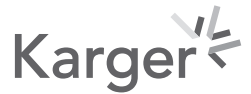


using SPSS software (v25, IBM SPSS Statistics for Windows, Armonk, NY, USA), and a $p$ value of $<0.05$ was statistically significant.

\section{Endovascular Treatment}

Prior to the therapeutic procedure, every patient underwent diagnostic 6-vessel angiography. All interventions were done under general anesthesia and weight-adjusted heparin application. Therefore, in most cases, a triaxial approach via a transarterial route was used including a guiding catheter (NeuronMax 6F, Penumbra, Alameda, CA, USA; Vista brite tip 8F, Cordis, Santa Clara, CA, USA), an intermediate catheter (Navien 054, Medtronic, Minneapolis, MN, USA; Neuron 070, Penumbra) and a DMSO and/or glue-compatible microcatheter (Apollo/Echelon, ev3, Irvine, CA, USA; Magic/Sonic, Balt, Irvine, CA, USA; Marathon, Medtronic, Minneapolis, MN, USA; Rapid Transit, Johnson \& Johnson, New Brunswick, NJ, USA; or Excelsior SL-10, Stryker, Fremont, CA, USA). In patients with transvenous access, a guiding catheter was placed in the jugular bulb via the femoral vein to enable embolization or balloon occlusion. Prior to embolization, a superselective angiogram of the feeding vessel via microcatheter was done to assess the fistula point, the extent of the shunt volume, and the pattern of venous flow. The choice of the embolic material and the amount of liquid (coils, liquid agents including Glubran 2 [GEM SRL, Viareggio, Italy], Magic Glue [BALT, Paris, France], Onyx [Medtronic], and SQUID [abmedica, Düsseldorf, Germany]) was left to the discretion of the operator. The general tenet of the treatment was normalizing the venous drainage with complete occlusion (CO) of the shunt. Different techniques were applied according to the angioarchitecture, flow profile, and accessibility of the DAVF. One approach was transarterial embolization with liquid agents only (NBCA and/or ethylene vinyl alcohol copolymer [EVOH]), or in combination with transvenous coil embolization of the affected sinus. Another combined strategy, that included EVOH and temporary balloon deployment, was an opportunity in individuals for whom sinus protection was essential due to involvement of the sinus in the venous drainage of the brain parenchyma. In all cases, the microcatheter was navigated via an arterial route as close as possible to the fistula point. If an acceptable proximity to the fistula point could not be reached, embolization with NBCA was the therapy of choice in this situation. This decision was based on the experience of the operator and was left to his/her discretion. If the liquid agents failed to cast into the venous side, in all patients with cavernous DAVFs and in nonsinus DAVFs, a venous approach was chosen. Shunt reduction was considered as the minimum objective of the first therapeutic session in symptomatic individuals or fistulas with CVR. Postoperative imaging was only performed if new neurological symptoms had occurred. Follow-up angiography was performed after the procedure, dependent on the initial presentation and the embolization result.

\section{Results}

\section{Baseline Characteristics and Treatment Approaches}

Overall, 61 patients with intracranial DAVFs were treated in our institution over 56 months. Eleven patients were excluded from further analysis due to pretreatment $(n=3)$, unavailable follow-up despite incomplete occlusion $(n=3)$, and ongoing therapy $(n=5)$. In the 50 remaining individuals, 93 treatments were performed (Table 1). The median age was 61 years (range 49-71 years) and 33 patients (66\%) were male. In 7 patients (14\%), the DAVF was an incidental finding on cerebral imaging. Symptomatic patients presented predominantly with pulsatile tinnitus $(17 / 50,34 \%)$ and ocular symptoms $(6 / 50,12 \%)$. Thirteen patients $(26 \%)$ presented with ICH. The DAVF was most frequently located at the transverse or sigmoid sinus $(20 / 50,40 \%$; Table 2; Fig. 1). Thirty-six fistulas (72\%) were classified as 


\section{Cerebrovascular Diseases Extra}

Table 1. Overall results

\begin{tabular}{|c|c|c|}
\hline \multicolumn{3}{|c|}{ Cerebrovasc Dis Extra 2020;10:84-93 } \\
\hline DOI: $10.1159 / 000509455$ & \multicolumn{2}{|c|}{$\begin{array}{l}\text { (c) } 2020 \text { The Author(s). Published by S. Karger AG, Basel } \\
\text { www.karger.com/cee }\end{array}$} \\
\hline \multicolumn{3}{|c|}{$\begin{array}{l}\text { Maus et al.: Endovascular Treatment of Intracranial DAVFs: A German Single-Center } \\
\text { Experience }\end{array}$} \\
\hline \multicolumn{2}{|l|}{ Number of patients } & 50 \\
\hline \multicolumn{2}{|l|}{ Number of treatments } & 93 \\
\hline \multicolumn{2}{|l|}{ Median age, years (IQR) } & $61(49-71)$ \\
\hline \multicolumn{2}{|l|}{ Male sex } & $33(66)$ \\
\hline \multicolumn{3}{|l|}{ Angiographic details } \\
\hline \multicolumn{3}{|l|}{ Type of DAVF } \\
\hline \multicolumn{2}{|l|}{ Transverse sigmoid } & $20(40)$ \\
\hline \multicolumn{2}{|c|}{ Carotid cavernous } & $5(10)$ \\
\hline \multicolumn{2}{|c|}{ Superior sagittal sinus } & $6(12)$ \\
\hline \multicolumn{2}{|c|}{ Tentorial } & $16(32)$ \\
\hline \multicolumn{2}{|c|}{ Ethmoidal } & $2(4)$ \\
\hline \multicolumn{2}{|c|}{ Superior petrosal sinus } & $1(2)$ \\
\hline \multicolumn{3}{|c|}{ Access } \\
\hline \multicolumn{2}{|l|}{ Transarterial } & $31(62)$ \\
\hline \multicolumn{2}{|c|}{ Transvenous } & $7(14)$ \\
\hline \multicolumn{2}{|c|}{ A combined approach } & $12(24)$ \\
\hline \multicolumn{2}{|c|}{ Venous use of balloon } & $3(6)$ \\
\hline \multicolumn{3}{|c|}{ Materials } \\
\hline \multicolumn{2}{|c|}{ Nonadhesive (Onyx/SQUID) only } & $14 / 50(28)$ \\
\hline \multicolumn{2}{|c|}{ Adhesive (Glubran/MagicGLUE) only } & $8 / 50(16)$ \\
\hline \multicolumn{2}{|c|}{ Coils only } & $4 / 50(8)$ \\
\hline \multicolumn{2}{|c|}{ A combination } & $24 / 50(48)$ \\
\hline \multicolumn{2}{|c|}{ Use of 1 material per patient } & $26(52)$ \\
\hline \multicolumn{2}{|c|}{ Use of $>1$ material per patient } & $24(48)$ \\
\hline \multicolumn{2}{|c|}{ Complete angiographic occlusion } & $46(92)$ \\
\hline \multicolumn{2}{|c|}{ Immediate final occlusion } & $28(56)$ \\
\hline \multicolumn{2}{|c|}{ Subsequent surgery } & $2(4)$ \\
\hline
\end{tabular}

Values express $n(\%)$, unless otherwise indicated.

"malignant," including 3 individuals with direct drainage into the spinal perimedullary veins (Cognard V; Fig. 2). The middle meningeal artery (MMA) and the occipital artery were the most frequently involved branches of the external carotid artery (70 and 46\%, respectively). An arterial route was chosen in 31 patients (62\%) and a combined approach in 12 (24\%); in the resulting 83 procedures, the most commonly targeted arteries were the MMA $(37 / 83,45 \%)$ and occipital artery $(14 / 83,17 \%)$. Multiple feeder arteries were present in 45 of 50 cases (90\%). Protection of the sinus with transvenous balloon catheter was done in 3/50 (6\%) patients. Nonadhesive precipitating EVOH (including Onyx and SQUID) and adhesive polymerizing NBCA were used in $46 / 50$ patients (92\%) as a single material or in combination.

\section{Angiographic Results and Clinical Outcome}

Complete occlusion of the DAVF was achieved in $48 / 50$ patients (96\%). Of those, 6 DAVFs $(12 \%)$ were downgraded and let to spontaneous thrombosis during follow-up. In 28/50 cases (56\%), only 1 procedure was performed to obtain CO. Two patients underwent subsequent surgery (due to anterior cranial fossa and tentorial DAVFs, respectively). In 2 patients, fistula recurrence occurred despite $\mathrm{CO}$ after the first procedure; both were treated successfully in a second intervention. In 36/50 patients (72\%), EVOH was used; 22 of these $36(61 \%)$ were treated in combination with NBCA or coils. EVOH only was used in 14/50 patients (28\%) with CO in every case. Of 19 patients treated initially with EVOH only, 12 showed an immediate CO (63\%), compared to $5 / 15$ patients $(33 \%, p=0.08)$ with CO after the primary use of NBCA. Among 93 procedures, the overall complication rate was 3\% (3 procedures) and included temporary cranial nerve palsy $(n=1)$, ischemic brain lesions due to carryover of embolic 
Table 2. Clinical and technical parameters according to DAVF location

\begin{tabular}{|c|c|c|c|c|c|c|}
\hline & $\begin{array}{l}\text { Transverse } \\
\text { sigmoid }\end{array}$ & $\begin{array}{l}\text { Carotid } \\
\text { cavernous }\end{array}$ & $\begin{array}{l}\text { Superior } \\
\text { sagittal }\end{array}$ & Tentorial & $\begin{array}{l}\text { Anterior } \\
\text { cranial fossa }\end{array}$ & $\begin{array}{l}\text { Superior } \\
\text { petrosal }\end{array}$ \\
\hline Number of patients & 20 & 5 & 6 & 16 & 2 & 1 \\
\hline Median age, years (IQR) & $62(47-69)$ & $55(43-74)$ & $53(51-59)$ & $63(55-72)$ & 48 (n.a.) & 75 (n.a.) \\
\hline Male sex & $14(70)$ & - & $5(83)$ & $13(81)$ & $1(50)$ & - \\
\hline \multicolumn{7}{|l|}{ Clinical characteristics } \\
\hline Tinnitus & $16(80)$ & - & - & $1(6)$ & - & - \\
\hline Headache & $3(15)$ & $2(40)$ & - & $1(6)$ & $1(50)$ & - \\
\hline Intracranial hemorrhage & $4(20)$ & - & $3(50)$ & $6(38)$ & - & - \\
\hline Seizure & $1(5)$ & - & $3(50)$ & - & - & - \\
\hline Exophthalmus & - & $3(60)$ & - & - & - & - \\
\hline Double vision & $1(5)$ & $2(40)$ & - & $1(6)$ & - & - \\
\hline Asymptomatic & $3(15)$ & - & - & $4(25)$ & $1(50)$ & - \\
\hline \multicolumn{7}{|l|}{ Angiographic details } \\
\hline “Malignant" DAVF (Cognard $\geq$ IIB) & $12(60)$ & $1(20)$ & $6(100)$ & $15(94)$ & $1(50)$ & $1(100)$ \\
\hline Multiple feeder & $17(85)$ & $5(100)$ & $5(83)$ & $16(100)$ & $1(50)$ & $1(100)$ \\
\hline \multicolumn{7}{|l|}{ Access } \\
\hline Transarterial & $10(50)$ & - & $5(83)$ & $13(81)$ & $2(100)$ & $1(100)$ \\
\hline Transvenous & $3(15)$ & $4(80)$ & - & - & - & - \\
\hline A combined approach & $7(35)$ & $1(20)$ & $1(17)$ & 3 (19) & - & - \\
\hline Number of necessary treatments & 28 & 8 & 13 & 40 & 3 & 1 \\
\hline Single treatment & $15(75)$ & $4(80)$ & $3(50)$ & $4(25)$ & $1(50)$ & $1(100)$ \\
\hline \multicolumn{7}{|l|}{ Materials } \\
\hline Onyx & $11(55)$ & - & $1(17)$ & $5(31)$ & $1(50)$ & - \\
\hline SQUID & $7(35)$ & $1(20)$ & $1(17)$ & $9(56)$ & - & $1(100)$ \\
\hline Coils & $8(40)$ & $4(80)$ & $1(17)$ & $4(25)$ & - & - \\
\hline Glubran & $7(35)$ & $1(20)$ & $5(83)$ & $11(69)$ & $1(50)$ & - \\
\hline MagicGLUE & - & - & - & $1(6)$ & - & - \\
\hline Use of 1 material per patient & $9(45)$ & $4(80)$ & $4(66)$ & $6(37)$ & $2(100)$ & $1(100)$ \\
\hline Complete angiographic occlusion & $20(100)$ & $5(100)$ & $6(100)$ & $13(81)$ & $1(50)$ & $1(100)$ \\
\hline
\end{tabular}

Values express $n(\%)$, unless otherwise indicated. n.a., not available.

material $(n=1)$, and temporary asystolia during DMSO injection prior to embolization $(n=$ 1). None of the patients had permanent deficits. Angiographic and clinical follow-up were available for $37 / 50(74 \%)$ and $41 / 50(82 \%)$ patients, respectively. The median follow-up period was 117 days (interquartile range [IQR] 90-161 days). In all patients that suffered initially from pulsatile tinnitus or ocular symptoms, complete recovery of the symptoms was observed due to complete fistula closure. The in-hospital mortality was $2 \%$ as 1 patient died due to baseline ICH. Procedure-related mortality was $0 \%$.

\section{Single versus Multiple Treatment Procedures}

A single treatment procedure was sufficient for $28 / 50$ patients (56\%) to achieve CO. Twenty-two patients (44\%) received multiple treatments, with an average of 3 procedures being necessary. For the occlusion of transverse sigmoid sinus DAVFs, a single treatment was more often sufficient than in the remaining population: $15 / 28$ (54\%) vs. 5/22 (23\%) ( $p=$ 0.04). However, for angiographic cure of tentorial DAVFs, multiple sessions were more often required: $12 / 22(55 \%)$ versus $4 / 28(14 \%)(p=0.0051)$. A CO after 1 procedure was achieved more often with EVOH than NBCA: 12/28 (43\%) versus 5/28 (18\%) ( $p=0.08)$. Otherwise, in the multiple treatment group, a combination of NBCA and EVOH was more frequently necessary with the single use of 1 of these agents: 17/22 (77\%) versus 5/22 (23\%) ( $p=0.0007)$. 


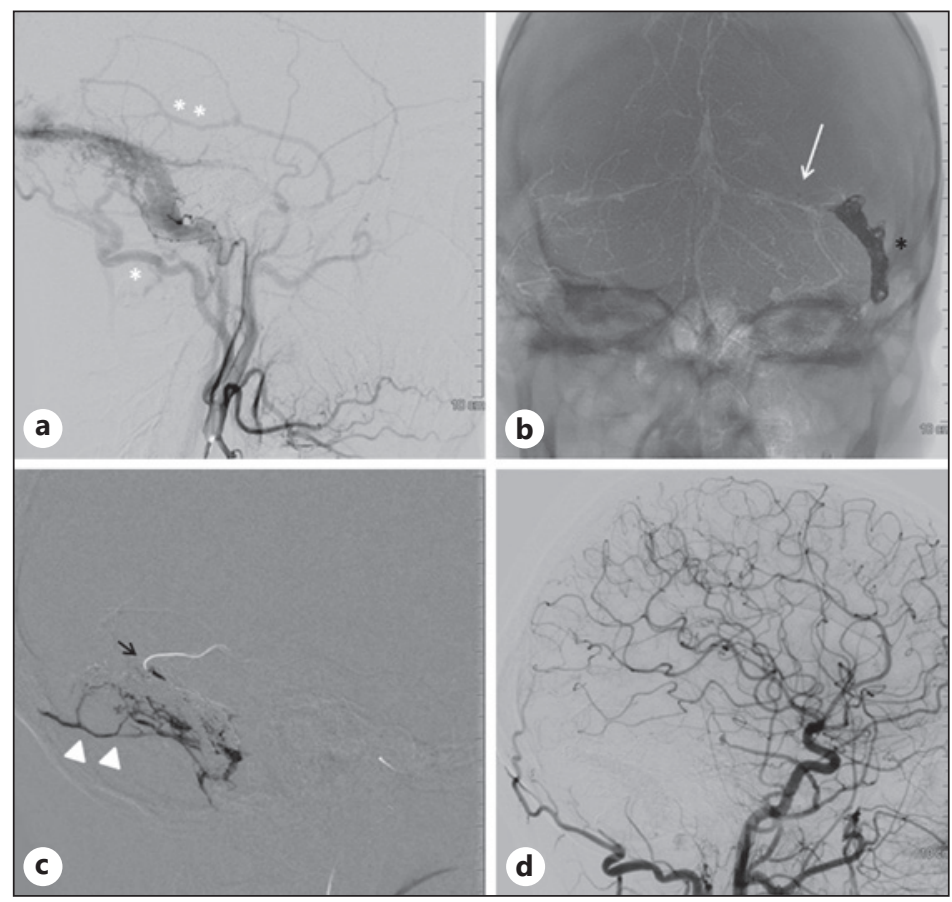

Fig. 1. a A 23-year-old patient presented with a left-sided pulsatile tinnitus due to a dural arteriovenous fistula (DAVF) with venous drainage into transverse-sigmoid sinus with retrograde flow (Cognard IIA). The fistula was supplied by multiple arterial feeder including the occipital artery (white asterisk) and middle meningeal artery (MMA, double asterisk). b As the affected sigmoid sinus was not involved in the regular venous drainage of the brain, transvenous sinus occlusion was the primary strategy. Therefore, embolization with coils (black asterisk) was performed with subsequent administration of Onyx via a second microcatheter that was initially navigated beyond the coil package (white arrow). c Since the fistula was not completely occluded, a microcatheter was navigated transarterially into the MMA close to the fistulous connection (black arrow) and $2 \mathrm{~mL}$ of Onyx were injected with favorable penetration of the fistula site and even retrograde casting of occipital branches (white arrowheads). d Final angiogram demonstrated complete occlusion of the DAVF. The patient was discharged 2 days after the procedure with complete resolution of symptoms.

\section{Discussion}

The results of our single-center series indicate that endovascular therapy of DAVF represents a safe, feasible, and effective approach which is associated with a high rate of complete fistula occlusion. In more than half of the patients, just 1 treatment procedure was sufficient to achieve CO, and all patients with pulsatile tinnitus and ocular symptoms were free of complaints at their follow-up visits.

The general tenet of endovascular treatment is a permanent closure of the DAVF and normalization of venous drainage with at least elimination of CVR. It needs to be considered that an arterial embolization of the fistula too close to the arteriovenous shunt should be avoided as persistent arterial flow might lead to the recruitment of small feeding arteries, with subsequent maintenance of blood supply to the fistula. Alternatively, an exclusive embolization of the venous site can increase the risk of venous occlusion and hypertension [14]. Based on the vascular geometry and the angioarchitecture of the DAVF, a transarterial or transvenous approach, or a combination of the two, can be chosen as the access of choice. Historically, transvenous embolization using coils and/or liquid agents has been the preferred approach. However, the occurrence of venous stenosis, sinus compartments, and complex 

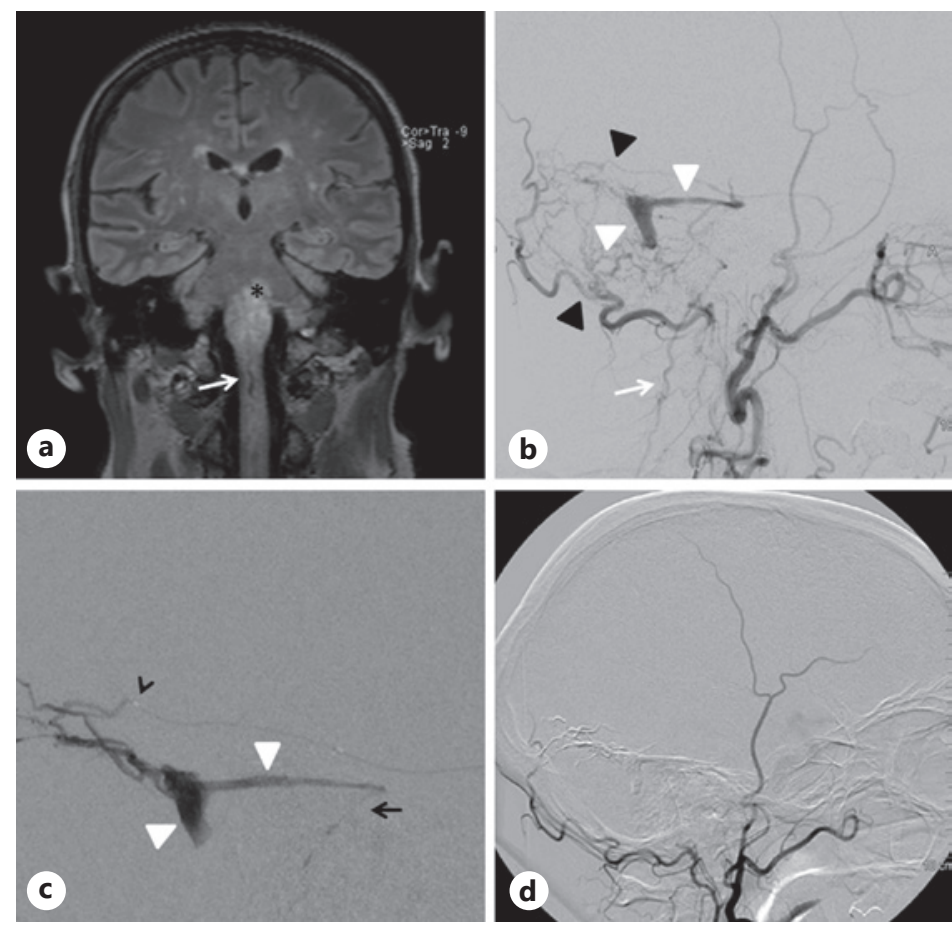

Fig. 2. a A 75-year-old patient was admitted to our hospital with sudden onset of parapalsy. Emergent magnet resonance imaging revealed a massive congestion edema of the brainstem (asterisk) with a dilated perimedullar vessel along the anterior surface of the spinal cord (white arrow). b One day later an angiography was performed under general anesthesia with evidence of an occipital dural arteriovenous fistula (DAVF). The DAVF was fed by branches of the middle meningeal artery and occipital artery (black arrowheads) with venous drainage into the proximal part of the sigmoid sinus, the superior petrosal sinus and subsequently into a perimedullar vein (white arrowheads). It is to mention that both the transverse sinus and internal jugular vein was occluded due to thrombosis. c A microcatheter was navigated via a transarterial access as close to the fistula point as possible (open arrowhead) followed by injection of SQUID with casting the fistulous connection, the affected sinus (white arrowheads) and the proximal part of the dilated vein (black arrow). $\mathbf{d}$ The DAVF was occluded immediately which was confirmed on follow-up angiogram 5 months after the procedure. The patient recovered during the initial hospital stay, with mild gait disturbances at clinical follow-up 18 months later.

venous anatomy reduces success probability via the transvenous route only. With the introduction and establishment of EVOH-based nonadhesive components during the last 2 decades, the treatment strategy has changed towards the transarterial approach (except for cavernous DAVFs), which requires superselective distal catheterization of the feeding pedicles for optimal delivery of the embolic materials. The use of such nonadhesive agents is nowadays well-established, with promising rates of CO. A recent study by Moenninghoff et al. [16] demonstrated a CO rate of $77 \%$ in patients exclusively treated with Onyx. Another recent multicenter study revealed a CO rate of $92 \%$ for different nonadhesive liquids [20]. This is in agreement with our experience for all patients treated exclusively with EVOH. Furthermore, the high efficacy of $\mathrm{EVOH}$ is underlined by the fact that $86 \%$ of these fistulas were occluded completely after 1 procedure in our cohort. We furthermore observed a trend that immediate $\mathrm{CO}$ rates with EVOH were higher than with NBCA, as described previously [21]. This can be explained by the advantages of $\mathrm{EVOH}$; it can be delivered more slowly with gradual precipitation due to its nonadhesive properties, thus allowing the casting of fistulous connections with better control than with NBCA ("plug-and-push") [4]. 
Maus et al.: Endovascular Treatment of Intracranial DAVFs: A German Single-Center Experience

In our study, a high portion of patients were treated repeatedly with a combination of different liquid embolic materials and/or coils. This can be explained by the high number of complex DAVFs in our cohort, with almost $75 \%$ of the patients having a CVR. In our study, $42 \%$ of the patients exhibited CVR with venous ectasia, as opposed to the studies of RangelCastilla et al. [22] and Johnson et al. [20], in which this subgroup comprised 14 and 22\%, respectively. Tentorial DAVFs, in particular, more often required multiple treatment sessions in our study, which may have been due to the extensive arterial supply and the location [23].

In patients with such complex fistulas, our in-house therapeutic objective is not primarily the immediate closure of the DAVF during 1 procedure, but rather a downstaging of the fistula with the elimination of CVR in a staged fashion, as radical embolization has been shown to be an independent predictor of complications [11]. We therefore pursue 2 different strategies which are dependent on the clinical presentation and angioarchitecture of the fistula. In severely affected patients (with neurological symptoms, venous congestion, or pulsatile tinnitus) or individuals with baseline ICH, our primary aim is to reduce the blood flow of the DAVF considerably, in order to improve the symptom level or decrease the risk of recurrent hemorrhage. Otherwise, it may be reasonable to target the smaller artery supply first; this causes the DAVF to rely on the remaining and large pedicles which are occluded in further procedures [4]. This approach might impede potential collateral recruitment and thereby reduce the rate of recurrence, which, at 4\%, was low in our study during follow-up.

The most commonly treated artery in our study was the MMA (45\%). This was due to the frequent involvement of the blood supply to the DAVF, and the anatomical conditions including feasible access and catheterization. The embolization of primary meningeal branches (e.g., the middle or posterior meningeal artery) might be a possible explanation for the high occlusion rate. Smaller involved arteries from other extracranial branches (e.g., the occipital or ascending pharyngeal artery) penetrate the skull via transosseous branches; this can be very tortuous with tiny vessel diameters. A distal positioning of the catheter close to the fistulous connection is frequently easier to achieve in the primary meningeal arteries which run along the inner side of the skull, with better penetration of embolic materials [4]. The choice of embolic agent was dependent on different characteristics including hemodynamic properties and accessibility. In some of the cases with high-flow shunts and in cases with microcatheter wedging, NBCA was primarily used. We used a transvenous approach primarily in cavernous and transverse-sigmoid DAVFs, as this access has been shown to be efficient for these fistula types $[13,24]$. In all patients, the cavernous sinus could be entered via the inferior petrosal sinus, and then completely occluded by means of coils. In half of the patients with a transverse-sigmoid DAVF, the sinus was either occluded or successfully protected by balloon catheters; the efficacy of transvenous treatment was previously reported as having an occlusion rate of $81 \%$ [13].

The complication- (3\%) and procedure-related (0\%) mortality rates in our cohort were acceptable and in line with previous studies $[11,16,19,25]$. To date, the largest case series on cranial DAVFs in the literature reported a complication rate of $7.7 \%$ and a mortality rate of $0.8 \%$ [11]. Although 2 patients were temporarily affected, no patients had permanent deficits. One patient temporarily exhibited facial nerve palsy after MMA embolization, a known phenomenon as the facial nerve is supplied by petrosal branches. Particular attention is given to potential anastomoses between the intra- and extracranial branches to avoid the carryover of embolic liquids. In 1 patient, a low amount of NBCA was transferred to the middle cerebral artery after embolization of the fistula pedicles arising from the maxillar artery. However, postinterventional MRI demonstrated minor ischemic lesions without any further clinical sequelae. In general, complications occurred in patients with DAVF location in the falcotentorial group (tentorial and anterior cranial fossa). This confirms the results of a recent study by Hiramatsu et al. [11] that demonstrated nonsinus-type location as a predictor of

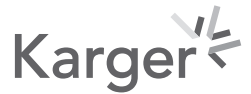


complications. Other studies also showed increased complication rates for the tentorial location in up to $31 \%$ [23].

A major limitation of this study was the retrospective design with the attendant selection bias, the absence of a control group, and the heterogeneity of the patient group, i.e., including patients with different clinical symptoms, DAVFs angioarchitecture, and location. The small sample size precludes further statistical analysis. However, as the study represents a "realworld" cohort, the clinical results and angiographic occlusion rates are promising and their validity is strengthened by a high rate of clinical and angiographic follow-up.

\section{Conclusion}

Endovascular treatment of intracranial DAVFs is feasible, safe, and effective with high rates of CO. In all patients, a complete recovery from pulsatile tinnitus or ocular symptoms was achieved due to complete fistula closure. In more than half of the patients, the DAVF was completely occluded after a single procedure. However, in tentorial DAVFs, multiple sessions were more often required.

\section{Statement of Ethics}

The study was conducted ethically in accordance with the World Medical Association Declaration of Helsinki. According to the guidelines of the respective local Ethics Committee, no approval was necessary for this anonymous retrospective study.

\section{Conflict of Interest Statement}

The authors have no conflicts of interest to declare.

\section{Funding Sources}

There was no funding of any research relevant to this study.

\section{Author Contributions}

V.M. data analysis and writing. F.D. data analysis. L.G. statistics and proofreading. A.W. data analysis and proofreading. W.W. idea, conceptual design, and proofreading. S.F. data analysis, writing, and proofreading.

\section{References}

1 Newton TH, Cronqvist S. Involvement of dural arteries in intracranial arteriovenous malformations. Radiology. 1969 Nov;93(5):1071-8.

2 Sarma D, ter Brugge K. Management of intracranial dural arteriovenous shunts in adults. Eur J Radiol. 2003 Jun;46(3):206-20.

3 Lasjaunias P, Chiu M, ter Brugge K, Tolia A, Hurth M, Bernstein M. Neurological manifestations of intracranial dural arteriovenous malformations. J Neurosurg. 1986 May;64(5):724-30. 
4 Vanlandingham M, Fox B, Hoit D, Elijovich L, Arthur AS. Endovascular treatment of intracranial dural arteriovenous fistulas. Neurosurgery. 2014 Feb; 74(Suppl 1):S42-9.

5 Ghobrial GM, Marchan E, Nair AK, Dumont AS, Tjoumakaris SI, Gonzalez LF, et al. Dural arteriovenous fistulas: a review of the literature and a presentation of a single institution's experience. World Neurosurg. 2013 Jul-Aug;80(1-2):94-102.

6 Cognard C, Gobin YP, Pierot L, Bailly AL, Houdart E, Casasco A, et al. Cerebral dural arteriovenous fistulas: clinical and angiographic correlation with a revised classification of venous drainage. Radiology. 1995 Mar; 194(3):671-80.

7 Borden JA, Wu JK, Shucart WA. A proposed classification for spinal and cranial dural arteriovenous fistulous malformations and implications for treatment. J Neurosurg. 1995 Feb;82(2):166-79.

8 Bulters DO, Mathad N, Culliford D, Millar J, Sparrow OC. The natural history of cranial dural arteriovenous fistulae with cortical venous reflux-the significance of venous ectasia. Neurosurgery. 2012 Feb;70(2):312-8.

9 Brown RD Jr, Wiebers DO, Nichols DA. Intracranial dural arteriovenous fistulae: angiographic predictors of intracranial hemorrhage and clinical outcome in nonsurgical patients. J Neurosurg. 1994 0ct;81(4):531-8.

10 Duffau H, Lopes M, Janosevic V, Sichez JP, Faillot T, Capelle L, et al. Early rebleeding from intracranial dural arteriovenous fistulas: report of 20 cases and review of the literature. J Neurosurg. 1999 Jan;90(1):78-84.

11 Hiramatsu M, Sugiu K, Hishikawa T, Nishihiro S, Kidani N, Takahashi Y, et al. Results of 1940 embolizations for dural arteriovenous fistulas: Japanese Registry of Neuroendovascular Therapy (JR-NET3). J Neurosurg. 2019 Jun:1-8.

12 Liebig T, Henkes H, Brew S, Miloslavski E, Kirsch M, Kühne D. Reconstructive treatment of dural arteriovenous fistulas of the transverse and sigmoid sinus: transvenous angioplasty and stent deployment. Neuroradiology. 2005 Jul; 47(7):543-51.

13 Kirsch M, Liebig T, Kühne D, Henkes H. Endovascular management of dural arteriovenous fistulas of the transverse and sigmoid sinus in 150 patients. Neuroradiology. 2009 Jul;51(7):477-83.

14 Santillan A, Nanaszko M, Burkhardt JK, Patsalides A, Gobin YP, Riina HA. Endovascular management of intracranial dural arteriovenous fistulas: a review. Clin Neurol Neurosurg. 2013 Mar;115(3):241-51.

15 Ambekar S, Gaynor BG, Peterson EC, Elhammady MS. Long-term angiographic results of endovascularly "cured" intracranial dural arteriovenous fistulas. J Neurosurg. 2016 Apr;124(4):1123-7.

16 Moenninghoff C, Pohl E, Deuschl C, Wrede K, Jabbarli R, Radbruch A, et al. Outcomes After Onyx Embolization as Primary Treatment for Cranial Dural Arteriovenous Fistula in the Past Decade. Acad Radiol. 2020 Jun; 27(6):e123-31.

17 Bank WO, Kerber CW, Cromwell LD. Treatment of intracerebral arteriovenous malformations with isobutyl 2-cyanoacrylate: initial clinical experience. Radiology. 1981 Jun;139(3):609-16.

18 Kerber C. Use of balloon catheters in the treatment of cranial arterial abnormalities. Stroke. 1980 Mar-Apr; 11(2):210-6.

19 Gross BA, Albuquerque FC, Moon K, McDougall CG. Evolution of treatment and a detailed analysis of occlusion, recurrence, and clinical outcomes in an endovascular library of 260 dural arteriovenous fistulas. J Neurosurg. 2017 Jun;126(6):1884-93.

20 Johnson CS, Chiu A, Cheung A, Wenderoth J. Embolization of cranial dural arteriovenous fistulas in the liquid embolic era: A Sydney experience. J Clin Neurosci. 2018 Mar;49:62-70.

21 Rabinov JD, Yoo AJ, Ogilvy CS, Carter BS, Hirsch JA. ONYX versus n-BCA for embolization of cranial dural arteriovenous fistulas. J Neurointerv Surg. 2013 Jul;5(4):306-10.

22 Rangel-Castilla L, Barber SM, Klucznik R, Diaz O. Mid- and long-term outcomes of dural arteriovenous fistula endovascular management with Onyx. Experience of a single tertiary center. J Neurointerv Surg. 2014 Oct; 6(8):607-13.

23 Byrne JV, Garcia M. Tentorial dural fistulas: endovascular management and description of the medial duraltentorial branch of the superior cerebellar artery. AJNR Am J Neuroradiol. 2013 Sep;34(9):1798-804.

24 Roy D, Raymond J. The role of transvenous embolization in the treatment of intracranial dural arteriovenous fistulas. Neurosurgery. 1997 Jun;40(6):1133-41.

25 Baltsavias G, Valavanis A. Endovascular treatment of 170 consecutive cranial dural arteriovenous fistulae: results and complications. Neurosurg Rev. 2014 Jan;37(1):63-71. 